\title{
States of matter inside the planets
}

\section{W.B. Hubbard}

Interiors of the Planets. By A.H. Cook. Pp.348. ISBN 0-521-23214-7. (Cambridge University Press: 1980.) $£ 25, \$ 59.50$.

Interiors of the Planets, a volume in the Cambridge Planetary Science series, is intended as a textbook for advanced students. The book reads rather more like an extended review article than a text, however; yet probably no other approach is possible in this rapidly-developing field.

A number of thorough, up-to-date accounts of important topics are scattered through the book. The initial discussion of the Earth and the derivation of interior models from seismic data and the AdamsWilliamson equation is lucid, as is the subsequent coverage of heat sources in the Earth and effects on the temperature gradient. The strongest parts of the book are those which deal with measurement of gravitational field parameters and with solid-body planetary motions - the use of artificial satellites, fly-bys and laser retroreflectors to determine gravitational field parameters and precession and libration constants is considered in some detail; and the first-order theory of figures, leading to the important Radau-Darwin approximation, is given in enough detail to make clear its physical significance.

There are two chapters on equations of state - separated by chapters on the Moon and terrestrial planets - a division which seems a little curious, since similar physical principles are involved. Nevertheless, the exposition of experimental techniques for measuring equations of state and phase transitions is a helpful guide to the intricate transformations which can occur in minerals at high pressure. The chapter on metallic and molecular hydrogen, which comes later, is also fairly complete, and gives the reader a good feeling for the convergence of very different methods for deriving pressure-density relations and other important thermodynamic parameters.

The chapter on the Moon is somewhat sketchy. Lunar seismology and the evidence for a lunar core from the moment of inertia are presented in useful detail, but an adequate discussion of important work on electromagnetic sounding of the lunar interior is lacking. The account of the formation of the lunar crust tends to be unclear - and many workers in this field would doubt the assertion that the crust may have accreted separately onto the Moon, from meteorites, after formation of the mantle.

The discussion of Mars, Venus and Mercury is likewise rather uneven. Moments of inertia are dealt with well, and the author succeeds in giving a clear, simple exposition of the relation between the moment of inertia and the relative effects of crust, polymorphic phase transitions and core. On the other hand, the effects of tidal dissipation are ignored, and the classic work of Goldreich and collaborators on spin-orbit coupling in terrestrial planets is mentioned only obliquely. Although some attention is given to the higher-order gravity fields of the Earth, Moon and Mars, a geoid map of each is needed for clarity; such a map is given for the Moon only, but without definition of units.

Similarly, the chapter on the Jovian planets has some fairly major gaps. Most discussions of the composition of this group start with the mass-radius diagram for self-gravitating spheres at zero temperature. The existence of a pronounced maximum radius for any such object at about the Jovian radius is convincing evidence for the preponderance of hydrogen in Jupiter and Saturn. Without such a diagram, the reader must take this important result on trust. In addition, the discussion of the heat balance of Jupiter and Saturn is misleading, and it is quite impossible for the solar wind or ultraviolet radiation to contribute in a significant way to the observed imbalance.

The author makes it clear that Interiors of the Planets is not a cosmogonically oriented book. His general theme is the use of established physical principles to draw general conclusions about the present interior state of planets. A synthesis of the derived interior structures of planets does require cosmogonical considerations, but we do not yet know enough to carry out such a synthesis.

The book will not suffice as a sole reference to planetary interiors for an interested student, but it will be a helpful addition to his library.

W.B. Hubbard is a Professor of Planetary Sciences at the University of Arizona.

\section{Logic in Galilean scholarship}

\section{A. Rupert Hall}

Galileo and the Art of Reasoning: Rhetorical Foundations of Logic and Scientific Method. By Maurice A. Finocchiaro. Pp.478. Hbk ISBN 90-2271094-5; pbk ISBN 90-227-1095-3. (Reidel: 1980.) Hbk Dfl.80, \$42; pbk Dfl.40, \$21.

THIS is, as befits the series in which it is published, very much a book by a philosopher for philosophers, a book about logic rather than about Galileo. A scientist, a historian or a general reader wishing to make an initial exploration of Galileo's style as a writer and a natural philosopher would do much better to sit down with an English version of Galileo's Dialogues on the Two Chief Systems of the World than to read this volume. Indeed, Professor Finocchiaro's study would be impossibly hard to follow for anyone who has neither familiarity with Galileo's book, to which it is directed, nor with contemporary scholarship in history and philosophy of science.

This is, in brief, a work of detailed and technical criticism, mostly of the logical structure of arguments offered either by Galileo himself or by would-be interpreters of Galileo, both historians and philosophers (and at least one great scientist Einstein). Finocchiaro exercises singular skill and acuity in the close analysis of reasoning in order to test whether opinions and generalizations purportedly justified by it are valid or otherwise: most commonly he finds them otherwise. Thus he finds grounds for strong reservations about statements or views relating to
Galileo put forward by Alexandre Koyré, Paul Feyerabend, Maurice Clavelin, Dudley Shapere, William Shea, Stillman Drake and many others. To give one example of the elaboration involved: a quotation from a book by Koyré published 42 years ago occupies half a page, its dissection 14 $1 / 2$ pages (admittedly of larger print). It is the fine structure of the Koyre passage that is faulted: "it would be unhistorical" the author concludes "to deny that the study of the history of science made great progress with Koyré: to turn the clock backwards is simply unthinkable".

It seems that his object is to make philosophical judgements upon the philosophical positions adopted by other writers on Galileo: Koyre's error lay in his being an apriorist rationalist; Feyerabend's in denying the rationality of Galileo's and modern science; Drake's in being an empiricist or perhaps totally aphilosophical, and so forth. This makes the road to a positive understanding of Galileo's reasoning long and hard. On the other hand, Finocchiaro does express plainly his sense of the merits of previous scholars, even though (in his view) all have made serious mistakes, and he has studied the Dialogue and the difficulty of translating it with exemplary care, providing very full analyses of both the content and the nature of the arguments used by Galileo.

There are, to me, paradoxes in Finocchiaro's book apart from the paradoxes of Feyerabend, the Zeno of out time. Why does he object to Koyré's writing that the Dialogue is "not a book of astronomy, nor even of physics. It is above 